\title{
BMJ Open Assessing the safety and feasibility of neoadjuvant hormone and radiation therapy followed by robot-assisted radical prostatectomy for treating locally advanced prostate cancer: protocol for an open-label, dose-escalation, single- centre, phase I clinical trial
}

\author{
Yu-Tian Xiao (D) , ' Xianzhi Zhao (D) , ${ }^{2}$ Yifan Chang, ${ }^{1}$ Xiaojun Lu, ${ }^{1}$ Ye Wang, ${ }^{1}$ \\ Huojun Zhang, ${ }^{2}$ Shancheng Ren ${ }^{1}$
}

To cite: Xiao Y-T, Zhao X, Chang $Y$, et al. Assessing the safety and feasibility of neoadjuvant hormone and radiation therapy followed by robot-assisted radical prostatectomy for treating locally advanced prostate cancer: protocol for an open-label, dose-escalation, single-centre, phase I clinical trial. BMJ Open 2020;10:e038678. doi:10.1136/ bmjopen-2020-038678

- Prepublication history for this paper is available online. To view these files, please visit the journal online (http://dx.doi org/10.1136/bmjopen-2020038678).

$\mathrm{Y}-\mathrm{TX}$ and $\mathrm{XZ}$ are joint first authors.

Received 19 March 2020 Revised 26 June 2020 Accepted 17 September 2020

Check for updates

(C) Author(s) (or their employer(s)) 2020. Re-use permitted under CC BY-NC. No commercial re-use. See rights and permissions. Published by BMJ.

For numbered affiliations see end of article.

Correspondence to Huojun Zhang; chyyzhj@163.com and Shancheng Ren; renshancheng@gmail.com

\begin{abstract}
Introduction Patients with locally advanced prostate cancer are at high risk of recurrence after definitive treatment. There are emerging data that radical prostatectomy can delay the progression of castration resistance and potentially prolong survival. Neoadjuvant radiation therapy improves local control and has shown survival benefit with favourable toxicity profiles in several other malignancies. We have designed this trial to investigate whether this combination, which theoretically maximises local control, is a safe and feasible approach for treating locally advanced prostate cancer.

Methods and analysis This study is a phase I, openlabel study to investigate the safety and feasibility of neoadjuvant hormone and radiation therapy followed by robot-assisted radical prostatectomy by a traditional $3+3$ dose-escalation design with four planned radiation dose levels (39.6 Gy/22F, 45 Gy/25F, 50.4 Gy/28F and 54 Gy/30F). Locally advanced prostate cancer patients with positive pelvic and/or retroperitoneal lymph nodes will be recruited. The primary objective is to determine the adverse events and maximal tolerable dose (MTD) of neoadjuvant radiotherapy. Toxicity will be assessed using the National Cancer Institute Common Toxicity Criteria V.5.0.

Ethics and dissemination This protocol was approved by the Institutional Review Board of Shanghai Changhai Hospital (ref. CHEC2019-070 and CHEC2019-082). The study will be performed in compliance with applicable local legislation and in accordance with the ethical principles developed by the World Medical Association in the Declaration of Helsinki 2013. Study results will be disseminated through conferences and peerreviewed scientific journals.

Trial registration numbers ChiCTR1900022716; ChiCTR1900022754.
\end{abstract}

\section{INTRODUCTION}

Prostate cancer is a major health problem worldwide, accounting for one fifth of newly
Strengths and limitations of this study

This protocol describes a phase I study with a traditional $3+3$ dose-escalation design.

- This study is expected to provide safety and feasibility profile to inform future prospective trials on preoperative radiotherapy in locally advanced prostate cancer.

- This study is monocentric, with relatively small sample size.

diagnosed malignancies in men. The number of prostate cancer patients in China has been continuously mounting and shows no sign at present of ceasing to rise, with approximately 99322 new diagnoses in the year 2018. ${ }^{1}$ Radical prostatectomy, commonly performed in a laparoscopic or robot-assisted approach, is a first-line curative treatment option for localised prostate cancer. ${ }^{2}$ Patients with locally advanced prostate cancer are at higher risk of recurrence, and the optimal treatment is still controversial. Current National Comprehensive Cancer Network and European Association of Urology guidelines all recommend radiation therapy plus long-term androgen deprivation therapy (ADT) as a primary treatment option. ${ }^{3} 4$ Increasingly, surgerybased multimodality treatment has become a feasible approach for treating high-risk localised and locally advanced prostate cancer. ${ }^{5}$ Whether individual patients may benefit from surgery remains to be elucidated, and a prospective phase III randomised controlled trial (RCT) comparing radical prostatectomy 
against radiation therapy and ADT for locally advanced prostate cancer patients is currently recruiting. ${ }^{6}$

However, there is evidence that patients might benefit from maximising local control with a combination of radiation therapy and surgery. Results from three phase III RCTs suggest improved biochemical progression-free survival and metastasis-free survival from immediate postoperative radiation therapy. ${ }^{7-9}$ We argue that similar survival benefits could be achieved through the use of radiation therapy and ADT in a neoadjuvant setting. Theoretically, the additional advantages of neoadjuvant radiation therapy include: (1) potential down-staging of the tumours, decreased rate of positive surgical margins and lower incidence of positive lymph nodes, (2) decreased hypoxia-induced radioresistance because of unaltered prostatic blood supply and (3) potential decrease in dosage and radiation-related toxicity. Indeed, the superiority of preoperative over postoperative chemoradiotherapy in terms of improved local control and reduced toxicity has been demonstrated by the phase III CAO/ ARO/AIO-94 study in locally advanced rectal cancer. ${ }^{10}$ In addition, given the considerable overlap of the radiation target volume, dose and schedule, the safety profile of preoperative radiotherapy for locally advanced prostate cancer and rectal cancer is roughly comparable. Therefore, we hypothesise that neoadjuvant radiation therapy is a safe and feasible approach for treating locally advanced prostate cancer.

\section{METHODS AND ANALYSIS}

\section{Study design}

This is a phase I, single-arm, single-centre observational study in Shanghai Changhai Hospital. The participants enrolled will be assigned to one of the four groups receiving $39.6 \mathrm{~Gy} / 22 \mathrm{~F}, \quad 45 \mathrm{~Gy} / 25 \mathrm{~F}, \quad 50.4 \mathrm{~Gy} / 28 \mathrm{~F}$ and $54 \mathrm{~Gy} / 30 \mathrm{~F}$ of preoperative radiation therapy plus ADT. A traditional $3+3$ dose escalation design will be utilised to determine the MTD of radiation therapy. Participants will then undergo robot-assisted radical prostatectomy and extended pelvic lymph node dissection (ePLND), followed by postoperative ADT for at least 2 years. The trial schedule is illustrated in figure 1. This trial protocol is structured and reported in accordance with the SPIRIT 2013 statement. ${ }^{1112}$

\section{Recruitment}

Patients who refer to the outpatient department of the trial site and meet the inclusion criteria will be recommended to participate in this trial by the physicians in charge of the study.

\section{Study participants}

Inclusion criteria

- Men between 18 and 75 years of age.

- Biopsy confirmed prostate adenocarcinoma without neuroendocrine differentiation, signet cell or small cell features.

- Locally advanced disease with positive pelvic lymph node (stage N1M0, ChiCTR1900022716) or positive retroperitoneal lymph node (stage M1a, ChiCTR1900022754), as determined by contrastenhanced CT, bone scan, MRI and/or prostatespecific membrane antigen (PSMA) targeted imaging (eg, 68Ga-PSMA PET/CT).

- Eastern Cooperative Oncology Group performance status $0-1$.

- An expected life expectancy of at least 5 years.

- Patients who are well informed of the current treatment options and willing to participate in the trial.

- Signed, written informed consent.

\section{Exclusion criteria}

A patient may not enter the study if ANY of the following applies:

- Lymph node metastases spreading beyond pelvic and retroperitoneal nodes.

- Presence of bone metastasis or distant organ metastasis.

- Prior exposure to any treatment for prostate cancer, including radiotherapy, chemotherapy, hormone therapy, focal therapy.

- Prior transurethral enucleation or resection of the prostate.

- Any abdominal surgery performed within 3 months prior to enrolment.

- Sustained use of anticoagulation and antiplatelet drugs.

- Any other previous or concurrent malignancies.

- Disease complicated by other severe systemic diseases which, in the judgement of the investigators, are likely

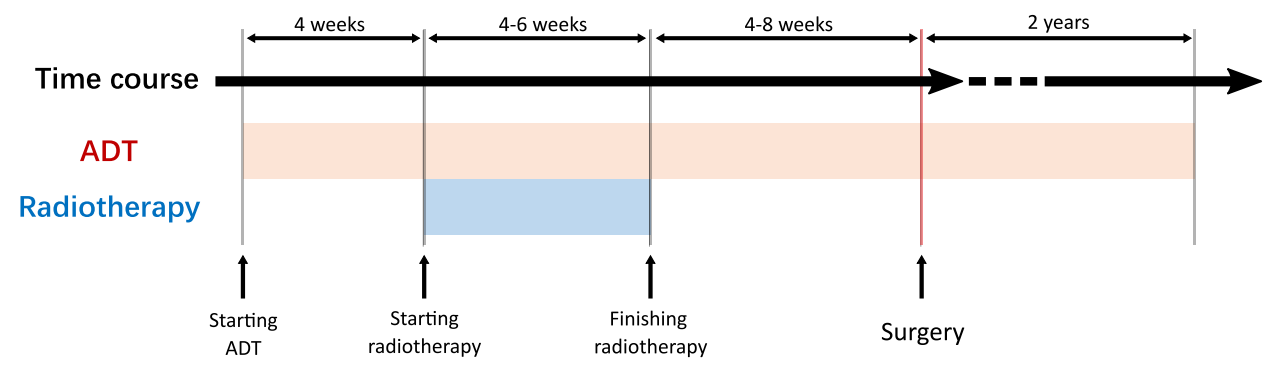

Figure 1 Schedule of the study. ADT, androgen deprivation therapy. 


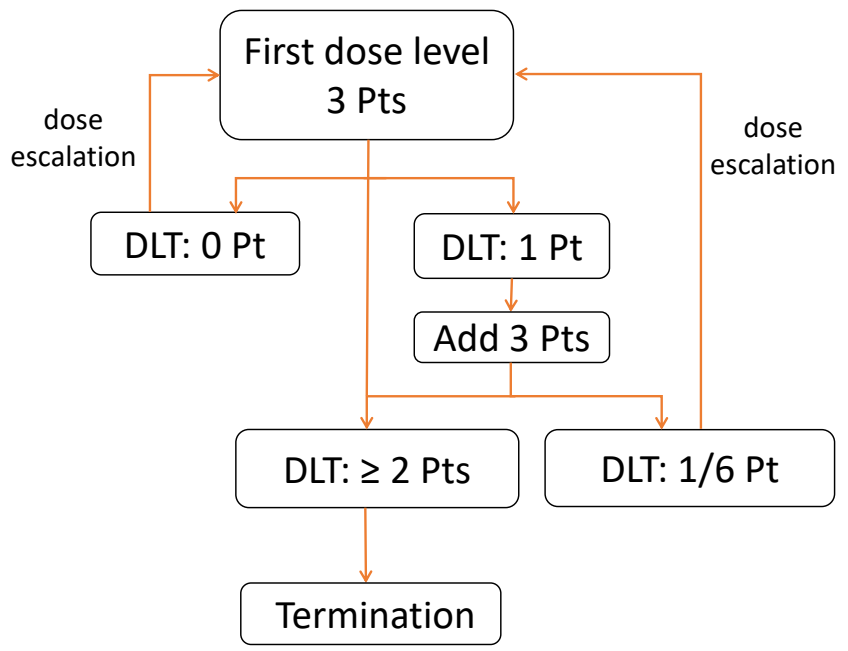

Figure 2 Graphical depiction of the 3+3 dose-escalation study design. DLT, dose-limiting toxicity. Pt, participant.

to interfere with the treatment, assessment or compliance associated with this trial.

- Participation in any other trial which is ongoing or has been completed within 3 months.

- Any contraindication for radiation therapy or surgery.

Dropout or suspension of the trial

- Occurrence of grade III/IV adverse events according to Common Terminology Criteria for Adverse Events (CTCAE) V.5.0.

- Requests from patients to withdraw from the trial.

- Lost to follow-up.

- Disease progression.

- Other potential situations that necessitate the termination of the trial.

\section{Interventions}

\section{Baseline evaluation}

Patients with histologically confirmed locally advanced prostate cancer who are eligible for this study will be evaluated for baseline characteristics. The evaluation will include demographics, medical history, concomitant diseases and medications, physical examination, vital signs, digital rectal examination, routine blood tests, high-resolution MRI of the pelvis, bone scan and $68 \mathrm{Ga}-$ PSMA PET/CT. Baseline characteristics of the included participants will be collected within 2 weeks prior to the initiation of ADT.

\section{Neoadjuvant radiation therapy plus ADT}

The ADT regimen for this trial includes bicalutamide $50 \mathrm{mg}$ PO once daily and goserelin acetate, a gonadotropin-releasing hormone agonist. The latter will be administered subcutaneously either at a dose of $3.6 \mathrm{mg}$ every 4 weeks, or at a dose of $10.8 \mathrm{mg}$ every 12 weeks.

Intensity modulated radiation therapy will be administered 4 weeks after the initiation of preoperative ADT. All patients shall undergo a contrasted CT simulation of the pelvis or abdomen of 5-mm-slice thickness in a supine position. The CT images will then be transferred to the treatment planning system for contouring the target volume and organs at risk (OARs) and planning. Critical normal structures include the small bowel, bladder, femoral head, rectum, spinal cord, prostatic urethra (if visualised), bulbous urethra, kidney. OARs shall be contoured according to the pelvic normal tissue contouring guidelines of Radiation Therapy Oncology Group (RTOG). ${ }^{13}$ This protocol offers dose guidelines to OARs based on prior published RTOG trials. ${ }^{14-16}$

The gross tumour volume (GTV) is contoured based on MRI. GTV includes prostate and seminal vesicle glands. GTV of the pelvic or retroperitoneal metastatic lymph node (GTVnd) is further confirmed by imaging. The clinical tumour volume (CTV) includes GTV, GTVnd, pelvic/retroperitoneal lymphatic drainage area. The superior border of the whole pelvis field extends to the L5-S1 interspace for N1 subgroup. The pelvic lymphatic drainage area includes bilateral total iliac lymph nodes, extra-iliac lymph nodes, intra-iliac lymph nodes, S1-S3 levels presacral lymph nodes and obturator lymph nodes. The superior border of the retroperitoneal field is $2-3 \mathrm{~cm}$ above the positive lymph nodes not exceeding renal artery level. The primary

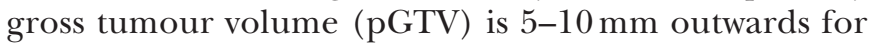
GTV in any direction, but only $5 \mathrm{~mm}$ in the posterior to reduce rectal irradiation. pGTVnd for GTVnd shall be delineated with an additional $5 \mathrm{~mm}$ margin and pCTV for CTV shall be delineated with an additional $5 \mathrm{~mm}$ margin separately.

Four radiation dose levels were planned: $39.6 \mathrm{~Gy} / 22 \mathrm{~F}$, $45 \mathrm{~Gy} / 25 \mathrm{~F}, 50.4 \mathrm{~Gy} / 28 \mathrm{~F}$ and $54 \mathrm{~Gy} / 30 \mathrm{~F}$. Radiation therapy will be delivered in 5 fractions per week. The initial two dose levels target whole pelvis/retroperitoneum, whereas in the latter two dose levels, a subsequent boost to the prostate, seminal vesicles and pelvic/retroperitoneal metastatic lymph nodes were added after reaching $45 \mathrm{~Gy}$.

\section{Dose escalation}

Dose escalation will be conducted in a $3+3$ design with dose levels of 39.6, 45, 50.4 and 54 Gy in 22, 25, 28 and 30 fractions, respectively. A traditional $3+3$ dose-escalation design will be adopted (figure 2). Briefly, three participants will initially be allocated into the starting dose cohort. If no dose-limiting toxicity (DLT) is observed in any of the three participants, the dose will be escalated and three new patients will be enrolled to receive the next level of radiation dose. If one participant develops any DLT, an additional three participants will be allocated into the same dose cohort. If there are multiple observations of DLT at any given dose level, the dose escalation will be stopped, and the previous dose level will be identified as the MTD. In this trial, DLT is defined as (1) any grade 4+toxicity, (2) any grade 3 toxicity except urinary incontinence, erectile dysfunction and responsive diarrhoea, (3) grade 2+fistula, (4) any grade colonic or rectal perforation or (5) any grade intraoperative rectal injury. 
Robot-assisted radical prostatectomy

Surgery will be scheduled within 4-8 weeks after the completion of radiation therapy, via a robot-assisted laparoscopic approach. ePLND will be performed. All surgical procedures will be performed by one single highly experienced robotic surgeon (SR).

\section{Postoperative treatment}

Participants will receive long-term post-operative ADT for at least 2 years. The regimen will remain the same as the pre-operative ADT regimen. Participants will be monthly evaluated for serum PSA and testosterone level at their local primary healthcare facilities. They will be followed up every 3 months for the first year and every 6 months for the following year. On tumour progression, salvage treatment including but not limited to abiraterone acetate/ prednisone treatment, chemotherapy and surgery, will be administered to the trial participants on documented progression in accordance with standard clinical practice.

\section{Outcomes and measurements}

The primary objective of this trial is to determine the adverse events and MTD of radiotherapy. Adverse events throughout the study will be assessed via CTCAE V.5.0 by research physicians or nurses.

Secondary endpoints include perioperative safety profile, efficacy of neoadjuvant treatment, rates of positive surgical margins, biochemical recurrence-free survival, overall survival and functional outcomes. Perioperative complications will be measured by Clavien-Dindo classification within 30 postoperative days. Continence will be measured by patient-reported pads used per day. Quality of life will be measured using Karnofsky Performance Status Scale, ${ }^{17}$ the Functional Assessment of Cancer Therapy-Prostate (V.4) instrument ${ }^{18}$ and the 5-level EQ-5D (EQ-5D-5L) instrument. ${ }^{19}$

\section{Determination of sample size}

The study is a dose-escalation study which implements a traditional 3+3 design with four dose levels. Three to six participants will be allocated to each dose level cohort. Therefore, the maximum per protocol sample size for this trial is 24 .

\section{Data management and monitoring}

The Institutional Review Board of Shanghai Changhai Hospital will monitor the reporting of adverse events and the quality of collected data on a semiannual basis. A planned interim analysis will be performed by the principal investigator when median postoperative follow-up reached 1 year.

\section{Statistical analysis}

All characteristics will be described by the frequency for classified variables, mean $\pm \mathrm{SD}$ and $95 \%$ CIs for normally distributed continuous data, and the median and range for non-normal distributional continuous data. Should any statistical hypothesis testing be used, a two-tailed test is preferred and the significance level threshold $(\alpha)$ is set as 0.05 . Statistical analyses will be performed using the $\mathrm{R}$ software V.4.0.0 or higher. $^{20}$

\section{Biological specimens}

Biological specimens acquired throughout the trial, including blood and tissue samples, will be stored for subsequent exploratory biomarker research. Informed consent of participants will be obtained prior to the acquisition of biological specimens.

\section{Patient and public involvement}

Patients or public have not been involved in the design of the present study.

\section{Ethics and dissemination}

This study was approved by the institutional review board of Shanghai Changhai Hospital (ref. CHEC2019-070 and CHEC2019-082). The study will be performed in compliance with applicable local legislation and in accordance with the ethical principles in the Declaration of Helsinki 2013. Eligible patients will be well informed of the purpose and schedule of this study. Written informed consent will be obtained by research physicians or nurses if patients decide to participate. All clinical data will be confidentially collected by research members. Findings of the study will be disseminated through publication in peer-reviewed scientific journals as well as relevant medical conferences.

\section{DISCUSSION}

The idea for maximising cancer local control originates from the 'seed and soil' hypothesis, which postulates that the growth of disseminating tumour cells is driven by factors secreted by the primary tumour. ${ }^{21}$ It has been demonstrated in metastatic prostate cancer that aggressive subclones persist in primary tumour site and can seed to metastatic lesions, leading to a vicious cycle of metastatic disease. ${ }^{22}{ }^{23}$ Furthermore, overall survival benefits can be observed in metastatic prostate cancer patients who have been treated with radiotherapy plus ADT compared with ADT alone. ${ }^{24}$ These data collectively suggest a role of maximising local control in the management of locally advanced and metastatic prostate cancer.

Currently, clinical trials on preoperative radiation therapy for prostate cancer have focused primarily on men with high-risk localised disease. To the best of our knowledge, there are two published modern-era trials that evaluated preoperative radiation therapy in localised prostate cancer. Koontz et al reported a phase I clinical trial in 12 men with high-risk localised prostate cancer who had completed long-course preoperative radiation therapy followed by radical prostatectomy. ${ }^{25}$ Radiation therapy was dose-escalated with dose levels of 39.6 $\mathrm{Gy} / 22 \mathrm{~F}, 45 \mathrm{~Gy} / 25 \mathrm{~F}, 50.4 \mathrm{~Gy} / 28 \mathrm{~F}$ and $54 \mathrm{~Gy} / 30 \mathrm{~F}$ in 5 fractions per week. The pelvic lymph nodes were treated up to $45 \mathrm{~Gy}$ with any additional dose given to the prostate and seminal vesicles. The superior border of the whole pelvis 
field extended to the L5-S1 interspace. Two patients developed urethral strictures requiring dilation. The reported 2-year biochemical recurrence-free survival was $67 \%$. Glicksman et al recently reported the long-term results of their phase I pilot study of 15 patients. ${ }^{26}$ Patients received $25 \mathrm{~Gy}$ in five consecutive daily fractions to the prostate only. At a median follow-up of 12.2 years, seven patients were free from biochemical relapse and six patients were metastasis-free. These results have motivated us to assess this treatment combination in locally advanced disease. Despite the limitations, the impact of our study has the potential to drive a paradigm shift in the management of locally advanced prostate cancer.

\section{Author affiliations}

${ }^{1}$ Department of Urology, Shanghai Changhai Hospital, Shanghai, China

${ }^{2}$ Department of Radiation Oncology, Shanghai Changhai Hospital, Shanghai, China

Acknowledgements We wish to acknowledge Dr Jin Fan (Department of Radiation Oncology, Fudan University Shanghai Cancer Centre) for providing support on protocol development.

Contributors $\mathrm{Y}-\mathrm{TX}, \mathrm{XZ}, \mathrm{YC}, \mathrm{HZ}$ and SR were involved in literature search, study conception, protocol development, conduct of the study and manuscript writing. $X L$ was involved in the conduct of the study. YW was involved in the conduct of the study and manuscript writing. SR is the principal investigator. $\mathrm{Y}-\mathrm{TX}, \mathrm{XZ}$ and $\mathrm{YC}$ are the trial coordinators. All authors contributed to and approved the final version of the manuscript.

Funding This research is partially funded by National Natural Science Foundation (81872105), National Major R\&D Program (2017YFC0908002) and Shanghai Changhai Hospital (2019YXK058).

Competing interests None declared.

Patient and public involvement Patients and/or the public were not involved in the design, or conduct, or reporting or dissemination plans of this research.

Patient consent for publication Not required.

Provenance and peer review Not commissioned; externally peer reviewed.

Open access This is an open access article distributed in accordance with the Creative Commons Attribution Non Commercial (CC BY-NC 4.0) license, which permits others to distribute, remix, adapt, build upon this work non-commercially, and license their derivative works on different terms, provided the original work is properly cited, appropriate credit is given, any changes made indicated, and the use is non-commercial. See: http://creativecommons.org/licenses/by-nc/4.0/.

\section{ORCID iDs}

Yu-Tian Xiao http://orcid.org/0000-0002-3039-8342

Xianzhi Zhao http://orcid.org/0000-0003-4096-3349

\section{REFERENCES}

1 Ferlay J, Ervik M, Lam F, et al. Global cancer Observatory: cancer today. Lyon, France: International Agency for Research on Cancer, 2018.

2 Chang SL, Kibel AS, Brooks JD, et al. The impact of robotic surgery on the surgical management of prostate cancer in the USA. BJU Int 2015;115:929-36.

3 Mottet N, van den Bergh RCN, Briers E, et al. EAU - ESTRO ESUR - SIOG Guidelines on Prostate Cancer 2018. Arnhem, The Netherlands: European Association of Urology Guidelines Office, 2018.

4 Mohler JL, Antonarakis ES, Armstrong AJ, et al. Prostate cancer, version 2.2019, NCCN clinical practice guidelines in oncology. J Natl Compr Canc Netw 2019;17:479-505.
5 Costello AJ. Considering the role of radical prostatectomy in 21st century prostate cancer care. Nat Rev Urol 2020;17:177-88.

6 Stranne J, Brasso K, Brennhovd B, et al. SPCG-15: a prospective randomized study comparing primary radical prostatectomy and primary radiotherapy plus androgen deprivation therapy for locally advanced prostate cancer. Scand J Urol 2018;52:313-20.

7 Bolla M, van Poppel H, Tombal B, et al. Postoperative radiotherapy after radical prostatectomy for high-risk prostate cancer: long-term results of a randomised controlled trial (EORTC trial 22911). Lancet 2012;380:2018-27.

8 Thompson IM, Tangen CM, Paradelo J, et al. Adjuvant radiotherapy for pathological T3NOMO prostate cancer significantly reduces risk of metastases and improves survival: long-term followup of a randomized clinical trial. J Urol 2009;181:956-62.

9 Wiegel T, Bottke D, Steiner U, et al. Phase III postoperative adjuvant radiotherapy after radical prostatectomy compared with radical prostatectomy alone in pT3 prostate cancer with postoperative undetectable prostate-specific antigen: ARO 96-02/AUO AP 09/95. J Clin Oncol 2009;27:2924-30.

10 Sauer R, Becker H, Hohenberger W, et al. Preoperative versus postoperative chemoradiotherapy for rectal cancer. N Engl J Med 2004;351:1731-40.

11 Chan A-W, Tetzlaff JM, Gøtzsche PC, et al. Spirit 2013 explanation and elaboration: guidance for protocols of clinical trials. BMJ 2013;346:e7586

12 Chan A-W, Tetzlaff JM, Altman DG, et al. Spirit 2013 statement: defining standard protocol items for clinical trials. Ann Intern Med 2013;158:200-7.

13 Gay HA, Barthold HJ, O'Meara E, et al. Pelvic normal tissue contouring guidelines for radiation therapy: a radiation therapy Oncology Group consensus panel atlas. Int J Radiat Oncol Biol Phys 2012;83:e353-62.

14 Michalski JM, Purdy JA, Winter K, et al. Preliminary report of toxicity following $3 \mathrm{D}$ radiation therapy for prostate cancer on $3 \mathrm{DOG} / \mathrm{RTOG}$ 9406. Int J Radiat Oncol Biol Phys 2000;46:391-402.

15 Michalski JM, Bae K, Roach M, et al. Long-Term toxicity following $3 \mathrm{D}$ conformal radiation therapy for prostate cancer from the RTOG 9406 phase I/II dose escalation study. Int J Radiat Oncol Biol Phys 2010;76:14-22.

16 Roach M, Winter K, Michalski JM, et al. Penile bulb dose and impotence after three-dimensional conformal radiotherapy for prostate cancer on RTOG 9406: findings from a prospective, multiinstitutional, phase I/II dose-escalation study. Int J Radiat Oncol Biol Phys 2004;60:1351-6.

17 Péus D, Newcomb N, Hofer S. Appraisal of the Karnofsky performance status and proposal of a simple algorithmic system for its evaluation. BMC Med Inform Decis Mak 2013;13:72.

18 Esper P, Mo F, Chodak G, et al. Measuring quality of life in men with prostate cancer using the functional assessment of cancer therapyprostate instrument. Urology 1997;50:920-8.

19 Luo N, Li M, Liu GG, et al. Developing the Chinese version of the new 5-level EQ-5D descriptive system: the response scaling approach. Qual Life Res 2013;22:885-90.

20 R Core Team. R: A language and environment for statistical computing. [program]. Vienna, Austria: R Foundation for Statistical Computing, 2020.

21 Psaila B, Lyden D. The metastatic niche: adapting the foreign soil. Nat Rev Cancer 2009;9:285-93.

22 Gundem G, Van Loo P, Kremeyer B, et al. The evolutionary history of lethal metastatic prostate cancer. Nature 2015;520:353-7.

23 Tzelepi V, Efstathiou E, Wen S, et al. Persistent, biologically meaningful prostate cancer after 1 year of androgen ablation and docetaxel treatment. J Clin Oncol 2011;29:2574-81.

24 Rusthoven CG, Jones BL, Flaig TW, et al. Improved survival with prostate radiation in addition to androgen deprivation therapy for men with newly diagnosed metastatic prostate cancer. J Clin Oncol 2016;34:2835-42.

25 Koontz BF, Quaranta BP, Pura JA, et al. Phase 1 trial of neoadjuvant radiation therapy before prostatectomy for high-risk prostate cancer. Int J Radiat Oncol Biol Phys 2013;87:88-93.

26 Glicksman R, Sanmamed N, Thoms J, et al. A phase 1 pilot study of preoperative radiation therapy for prostate cancer: long-term toxicity and oncologic outcomes. Int J Radiat Oncol Biol Phys 2019;104:61-6. 